YITP-01-33

April 2001

\title{
Implications of Muon anomalous magnetic moment for Direct detection of Neutralino Dark Matter
}

\author{
Yeong Gyun Kim and Mihoko M. Nojiri \\ YITP, Kyoto University, Kyoto, 606-8502, Japan
}

\begin{abstract}
We investigate the implications of the recent measurement of muon anomalous magnetic moment for the direct detection of neutralino dark matter in the three different SUSY models: mSUGRA, a model with non-universal Higgs mass, and an $S O(10)$ GUT model. We consider two extreme scenario for $\Delta a_{\mu}$ bound, i.e. $27 \times 10^{-10}<\Delta a_{\mu}<59 \times 10^{-10}$ ( $1 \sigma$ bound) and $0<\Delta a_{\mu}<11 \times 10^{-10}$ ( $2 \sigma$ below). In mSUGRA model, the counting ratio may be above the sensitivity of the future experiments when parameters are within $1 \sigma$ bound of $\Delta a_{\mu}$. However, the $\Omega_{\chi}$ tends to be high compared to the currently favored value $\Omega=0.3$. For models with the non-universal scalar masses, the possibility to have the consistent $\Omega_{\chi}$ and the high counting ratio is open up in the region of parameter space where Higgsino mass $\mu$ is smaller than mSUGRA prediction. In particular, in the $S O(10)$ model, the LSP dark matter detection rate may be enhanced by almost one order of magnitude compared to mSUGRA and the model with non-universal Higgs mass, for cosmologically acceptable $\Omega_{\chi} h^{2}$. The highest detection rate of LSP dark matter occurs in the region where the LSP constitutes a subdominant part of local halo DM. Implication of SUSY mass parameter measurement under the cosmological constraint is also discussed.
\end{abstract}




\section{Introduction}

The Minimal Supersymmetric Standard Model (MSSM) [1] is one of the best motivated extensions of the Standard Model. It offers a natural solution of the hierarchy problem [2] as well as amazing gauge coupling unification [3]. Since naturalness requires that at least some superparticles have masses at or below the $\mathrm{TeV}$ scale, supersymmetric theories generally predict a rich phenomenology at future colliders such as Tevatron, LHC, or proposed linear colliders (LC) [由]. As an extra "bonus", the simplest version of the MSSM, where $R$-parity is conserved, also contains a new stable particle (the lightest supersymmetric particle, LSP); in most cases this is the lightest neutralino, which often makes a good Dark Matter(DM) candidate [5].

Though we might have to wait for LHC experiment to start for the direct discovery of supersymmetry, we may be able to probe SUSY models earlier by using precision measurements in low energy experiments. Sparticles contribute to low energy measurements through loop effects, and these effects may become significant if the their masses are not too large. Another possibility is direct detection of dark matter by the detector placed deep underground [6, 7, 8, 9, 10. Note that the existence of dark matter already indicates the needs of new physics.

Recently the Brookhaven E821 experiment has released a measurement of the muon anomalous magnetic moment, reporting a 2.6 $\sigma$ deviation from the standard model value [11]

$$
\Delta a_{\mu} \equiv a_{\mu}^{e x p}-a_{\mu}^{S M}=43(16) \times 10^{-10}
$$

which has generated considerable interest. This deviation may indeed be a sign of new physics beyond the standard model (SM) and could be accommodated by supersymmetric contributions [12]-26]. There are essentially two types of diagrams involving superparticles which contribute to $a_{\mu}$, i.e., neutralino-smuon and chargino-sneutrino loop diagrams [27]. Since $a_{\mu}$ requires chirality violation, for $\tan \beta \gg 1$ the dominant contributions are proportional to the product of an electroweak gauge coupling and the Yukawa coupling of the muon, where the latter factor either comes directly from the higgsino component of the chargino or neutralino in the loop, or from $\tilde{\mu}_{L}-\tilde{\mu}_{R}$ mixing. For $\tan \beta \gg 1$ one has thus $\Delta a_{\mu} \propto \tan \beta$. If mass splittings between different sparticles are not too large, so that their mass scale can be described by the single parameter $m_{\mathrm{SUSY}}$, the overall result can be estimated as [28]

$$
\left|\Delta a_{\mu}\right|=\frac{1}{32 \pi^{2}}\left(\frac{5}{6} g_{2}^{2}+\frac{1}{6} g_{1}^{2}\right) \frac{m_{\mu}^{2}}{m_{\mathrm{SUSY}}^{2}} \tan \beta
$$

where $g_{1}$ and $g_{2}$ are the $U(1)_{Y}$ and $S U(2)$ gauge couplings, respectively. In our convention, the sign of $\Delta a_{\mu}$ is equal to the sign of Higgsino mass parameters $\mu$, when the gaugino masses and $\tan \beta$ are positive. Therefore $\Delta a_{\mu}$ imposes non-trivial constraints on the MSSM parameters such as the sign of $\mu$, superparticle masses and the ratio $\tan \beta$ of the vacuum expectation values (vevs) of the two neutral Higgs fields of the MSSM. The new data prefers a positive $\mu$ and one has an upper or lower limit on the chargino and sneutrino masses for a given $\tan \beta$. Through these non-trivial constraints, $\Delta a_{\mu}$ will affect the predictions of other observables.

There exist relations between the SUSY contribution to $a_{\mu}$ and the predicted LSP-nucleon scattering cross section [29]. A direct connection comes from the common dependence on $\tan \beta$, since both $a_{\mu}$ and $\sigma_{\chi p}$ increase with increasing $\tan \beta$. Similar to the SUSY contribution to $a_{\mu}$, the leading contributions to the spin-independent (coherent) contribution to $\sigma_{\chi p}$ also involve violation of chirality [30]. Main contributions come from the exchange of $\mathrm{CP}$-even Higgs bosons. It couples to the strangeness component of proton or neutron, whose coupling has the same $\tan \beta$ dependence as the Yukawa coupling of the muon. Note also that the sign of $\mu$, favored by $a_{\mu}$ measurement, suggests large value of $\sigma_{\chi p}$ for smaller LSP mass due to the 
lack of accidental cancellation of couplings [29]. Importance of DM search experiments have now increased substantially.

In general, larger $\sigma_{\chi p}$ implies smaller relic DM density $\Omega_{\chi} h^{2}$. Since the Higgs-LSP-LSP couplings require higgsino-gaugino mixing, they scale like $1 / \mu$ for $\mu^{2} \gg M_{Z}^{2}$. The Higgs exchange contribution to $\sigma_{\chi p}$ then scales like $1 / \mu^{2}$. On the other hand, the change in the size of $\mu$ also affects $\Omega_{\chi} h^{2}$ through the LSP dark matter annihilation into gauge bosons or s-channel Higgs boson. The annihilation cross section for $\chi \chi \rightarrow W W$ and $Z Z$ are highly suppressed in the bino-like LSP case which is favored in mSUGRA model. However, a large cross section is possible when the LSP has a significant Higgsino component. This is because $W W$ cross section depends on $W \chi \chi^{ \pm}$interactions and only couplings $W \tilde{H}^{0} \tilde{H}^{ \pm}$and $W \tilde{W}^{0} \tilde{W}^{ \pm}$ are allowed by gauge invariance. For $Z Z$ cross section, it depends on $Z \chi \chi_{i}^{0}$ interactions which is possible only through $Z \tilde{H}^{0} \tilde{H}^{0}$ couplings. Hence a reduction of $\mu$ (an increase in higgsino component of the LSP) implies an increase of LSP annihilation cross section, which in turn gives a reduction of $\Omega_{\chi} h^{2}$. The pair annihilation into fermions through s-channel pseudoscalar Higgs boson is also enhanced if LSP is the mixture of gaugino and higgsino.

The relations between $a_{\mu}$ and $\sigma_{\chi p}$, and $\sigma_{\chi p}$ and $\Omega_{\chi} h^{2}$ we discussed so far are model independent. One may further investigates more model dependent aspect of the relation. This has been intensively studied in a class of model called mSUGRA with an universal scalar mass $m$, an universal gaugino mass $M$, and an universal trilinear coupling $A$ [29, 20, 21, 26]. The model relates a lower bound of higgs mass and $\Delta a_{\mu}$ to the constraints to right handed slepton and LSP masses, and a simple flavor structure of soft masses leads the prediction to $b \rightarrow s \gamma$.

The parameter space of mSUGRA is then quite limited. This is because the model predicts $\mu \gg M_{1}$ unless $\tan \beta \gg 1$ or $m \gg M$. This tends to lead to too large a $\Omega_{\chi} h^{2}$, because the LSP pair annihilation cross section, $\sigma_{\chi p}(\chi \chi \rightarrow X)$ is proportional to $m_{\chi}^{2} / m_{\tilde{l}_{R}}^{4}$ while Higgs mass and $a_{\mu}$ constraints give a lower bound of those particles masses in general. The $b \rightarrow s \gamma$ constraint also pushes up the overall SUSY scale. Especially for large $\tan \beta(\sim 30)$, one might need stau-neutralino coannihilation or a s-channel annihilation of the neutralino to make relic DM density reasonable [31]. The DM signal also turns out to be rather low; the maximal value of the cross section is only slightly above the proposed sensitivities.

Note however that the constraint strongly relies on the assumption that all scalar masses are universal at GUT scale. The rather parameter independent prediction $\mu \gg M$ in mSUGRA is actually relying on the strict universality. Smaller value of $\mu$ can be easily achieved in a model with non-universal Higgs masses [29]. This leads to consistent DM density and larger counting ratios. The constraint from $b \rightarrow s \gamma$ is sensitive to on the structure of scalar masses.

A study of non-mSUGRA model may be useful in this stage where the experimental constraints start to strongly limit the parameter space of mSUGRA. Given the upper limit $\sigma_{\chi p}$ in mSUGRA, discovery of DM in the early stage of CDMS II experiment would enforce us to face to more flexible models. In this paper, we investigate the implications of recent measurement of muon anomalous magnetic moment for direct detection of Neutralino Dark Matter [32, 30, 33] in the various SUSY models. We impose the accelerator bounds of Higgs and sparticle masses. However, the prediction for $b \rightarrow s \gamma$ decay rate is sensitive to details of the flavor structure of the soft breaking terms, unlike the quantities we consider here. We therefore do not attempt to analyze the constraint from $b \rightarrow s \gamma$ decays quantitatively .

The muon anomalous magnetic moment will be further checked using four times more data collected already. On the other hand, it can be consistent with the standard model value if one takes into account other theoretical calculations of the hadronic vacuum polarization 35.

\footnotetext{
${ }^{1}$ In mSUGRA, the constraint from $b \rightarrow s \gamma$ decay rate is not important compared to that from Higgs mass for $\mu>0$ case [34].
} 
In this regards, we consider two extreme cases for the range of $\Delta a_{\mu}$;

$$
27 \times 10^{-10}<\Delta a_{\mu}<59 \times 10^{-10} \quad(1 \sigma \text { bound })
$$

and

$$
0<\Delta a_{\mu}<11 \times 10^{-10} \quad(2 \sigma \text { below })
$$

We study the minimal supergravity (mSUGRA) model as well as some other SUSY models where the assumption of strict scalar mass universality at the GUT scale is relaxed. Unlike mSUGRA, the relic density of neutralino could be very small for those models. Since the direct DM detection rate depends on the product of the $\sigma_{\chi p}$ and local LSP density $\rho_{\chi}$, one must consider what fraction of our local halo density $\rho_{\text {local }}$ could be composed of neutralinos. Unless there is separation for different types of DM, the ratio of LSP DM to total DM should be the same locally in the Galaxy and globally in the whole Universe. In our paper we present the thermal relic density $\Omega_{\chi}$ and $\sigma_{\chi p}$ separately. However, following assumption might be useful to estimate the signal ratio,

$$
\rho_{\chi}=\rho_{\text {local }} \times\left(\frac{\Omega_{\chi}}{\Omega_{D M}}\right)
$$

where $\Omega_{D M}$ is the total contribution of DM to the total energy density of the Universe. When combined with the reduced Hubble constant $h \simeq 0.7$ and $\Omega_{D M} \simeq 0.3$ we have $\Omega_{D M} h^{2} \simeq 0.15$. For our present study, we assume that $\rho_{\chi}=\rho_{\text {local }}$ if $\Omega_{\chi} h^{2} \geq 0.15$, and present the contours of future experimental reach using local LSP density rescaled as $\rho_{\chi}=\rho_{\text {local }} \times\left(\Omega_{\chi} h^{2} / 0.15\right)$ if $\Omega_{\chi} h^{2}<0.15$. For convenience, we define the scaled cross section,

$$
\begin{aligned}
\sigma_{\text {scaled }} & \equiv \sigma_{\chi p} \times\left(\Omega_{\chi} h^{2} / 0.15\right) & \text { for } \Omega_{\chi} \mathrm{h}^{2}<0.15 \\
& \equiv \sigma_{\chi p} & \text { for } \Omega_{\chi} \mathrm{h}^{2} \geq 0.15
\end{aligned}
$$

We will find that the increase in the $\sigma_{\chi p}$ may compensate the decrease in the $\Omega_{\chi} h^{2}$ so that the DM detection rate more or less remains unchanged 9 . For some model we also see that more than one order of magnitude enhancement of the counting ratio is possible when LSP is the subdominant component of the local halo.

\section{2 mSUGRA}

In the minimal supergravity model, it is usually assumed that all squared scalar masses receive a common soft SUSY breaking contribution $m_{Q}^{2}=m_{U}^{2}=m_{D}^{2}=m_{L}^{2}=m_{E}^{2}=m_{H_{u}}^{2}=m_{H_{d}}^{2} \equiv$ $m^{2}$ at the GUT scale $M_{X} \simeq 2 \cdot 10^{16} \mathrm{GeV}$, while all gauginos receive a common mass $M$ and all trilinear soft terms unify to $A$ at the same scale. The renormalization group (RG) evolution of soft breaking squared Higgs masses then leads to consistent breaking of the electroweak symmetry, provided the higgsino mass parameter $\mu$ can be tuned independently [37]. In this paper, we choose the weak scale input parameters $m_{b}\left(m_{b}\right)=4.2 \mathrm{GeV}, m_{t}\left(m_{t}\right)=165 \mathrm{GeV}$, and $\tan \beta$. We minimize the tree level potential at renormalization scale $Q=\sqrt{m_{\tilde{t}} m_{t}}$, which essentially reproduces the correct value of $\mu$ obtained by minimizing the full 1 -loop effective potential [38]. With these assumptions, the mSUGRA model allows four continuous free parameters $(m, M, A$ and $\tan \beta)$. We take $\mu>0$ because of BNL constraint.

\footnotetext{
${ }^{2}$ The model independent situation is recently discussed in [36].
} 

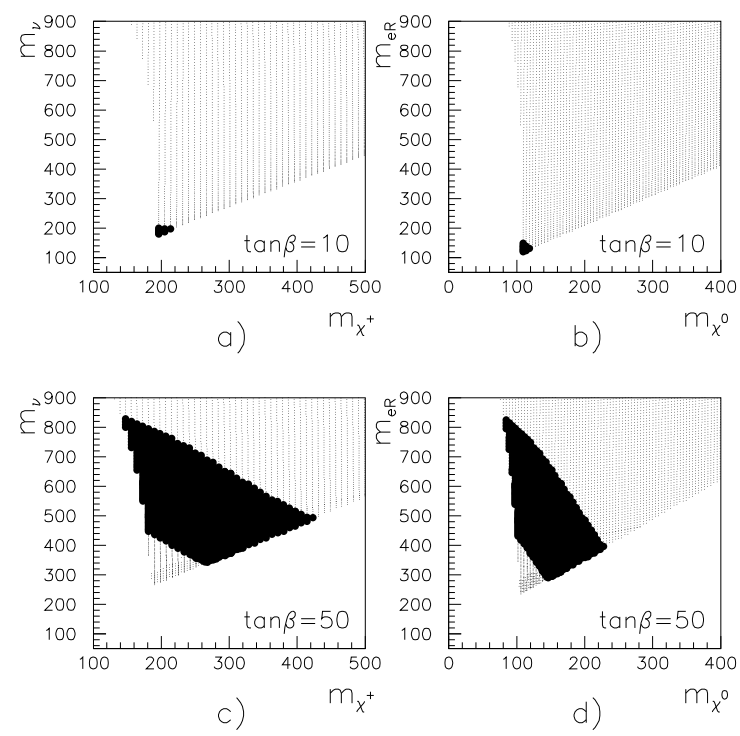

Figure 1: Allowed mass ranges of (a) chargino-sneutrino and (b) LSP-SU(2) singlet slepton in mSUGRA for $\tan \beta=10 . \tan \beta=50$ case is shown in (c) and (d). We take $A=0$ and $\mu>0$, and scan $m \in[0,1] \mathrm{TeV}$ and $M \in[0,1] \mathrm{TeV}$, subject to experimental constraints. The heavily marked points satisfy $1 \sigma$ bound of $\Delta a_{\mu}$.

For generic SUSY parameters, the chargino-sneutrino diagram provides a dominant contribution to $a_{\mu}$. In Fig. 1 (a) and (c), we plot the allowed mass range of chargino and sneutrino for two different choices of $\tan \beta=10$ and 50 respectively. The allowed mass range of LSP and $\mathrm{SU}(2)$ singlet sleptons are shown in Fig. 1 (b) and (d) for $\tan \beta=10$ and 50 respectively. Here, we take $A=0$ and $\mu>0$ and allow $m$ and $M$ to vary in the intervals $m<1 \mathrm{TeV}$ and $M<1 \mathrm{TeV}$. For the parameter range scanned in this plot, we find that the lightest Higgs boson $h$ couples essentially like the single Higgs boson of the SM; we thus demand that its mass $m_{h}>111 \mathrm{GeV}$. This follows from recent LEP results [39], allowing for a $2 \mathrm{GeV}$ theoretical uncertainty in the calculation of $m_{h}$ [40]. Further uncertainty on $m_{h}$ comes from the error of top quark mass value $\left(m_{h}\right.$ is varied by $\sim \pm 3 \mathrm{GeV}$ when $m_{t}$ is varied by $\pm 5 \mathrm{GeV}$ around $175 \mathrm{GeV}$ [1]). We include loop corrections to the masses of neutral Higgs bosons from the third generation quarks and squarks, including leading two-loop corrections [40]. We further require that the chargino mass $m_{\tilde{\chi}_{1}^{+}}>100 \mathrm{GeV}$ 42]. We exclude regions where the LSP is charged, i.e., $m_{\tilde{\tau}_{1}}<m_{\tilde{\chi}_{1}^{0}}$ or $m_{\tilde{t}_{1}}<m_{\tilde{\chi}_{1}^{0}}$. The parameter region is cut by Higgs mass constraint at left edge of the allowed region, and the lower edge of the allowed region is determined by neutral LSP constraint. The heavily marked points satisfy the further requirement of $27 \times 10^{-10}<\Delta a_{\mu}<59 \times 10^{-10}$. We use the expressions of ref. [28] for the calculation of $\Delta a_{\mu}$.

For $\tan \beta=10$, only small range of sparticle masses $\left(m_{\chi^{ \pm}} \sim 200 \mathrm{GeV}\right.$ and $\left.m_{\tilde{\nu}} \sim 200 \mathrm{GeV}\right)$ are allowed by the lightest Higgs mass bound, the requirement of the neutral LSP and $1 \sigma$ bound of $\Delta a_{\mu}$. For the small $\tan \beta$ values, the lower bound of $\Delta a_{\mu}$ gives a strict upper limit on sparticle masses because $\Delta a_{\mu}$ is proportional to $\tan \beta$ as mentioned already. On the other hand, the lightest Higgs mass bound $m_{h}>111 \mathrm{GeV}$ requires lower limit of sparticle masses because large radiative corrections from top and stop loops are required. These two opposite tendency allow only limited region of parameter space for the small $\tan \beta$ values. 
Upper limit of sparticle masses increase as $\tan \beta$ increases. For $\tan \beta=50$, the maximal values of light chargino and sneutrino mass are $\sim 430 \mathrm{GeV}$ and $\sim 820 \mathrm{GeV}$ respectively within $1 \sigma$ bound of $\Delta a_{\mu}$. For large $\tan \beta$ values, the region of parameter space where $m_{\chi^{ \pm}}, m_{\tilde{\nu}}$ is small is excluded by the upper bound of $\Delta a_{\mu}$ measurement because small sparticle masses gives too large $\Delta a_{\mu}$ values in this case.

These upper (and lower) bounds of sparticle masses with the fact that $\mu>0$ is preferred has a significant importance on the predictions of neutralino-proton cross section, $\sigma_{\chi p}$ [29, 21] and neutralino dark matter relic density, $\Omega_{\chi} h^{2}$. In most situations the dominant contribution to the spin independent amplitude is the exchange of the two neutral CP-even Higgs bosons. The sizes of the $\tilde{\chi}_{1}^{0} \tilde{\chi}_{1}^{0}(h, H)$ couplings do depend quite significantly on the sign of $\mu$, unless $\tan \beta \gg 1$ [43]. In particular, for $\mu<0$ strong cancellations occur [30] both within different contributions to the same coupling, and between the $h$ and $H$ exchange contributions to $\sigma_{\chi p}$. Because positive $\mu$ is preferred by $\Delta a_{\mu}$ experiment, cancellations would not occur. Also the upper (lower) limit of sparticle masses has a significant effect on the minimum (maximum) values of neutralino-proton cross section and neutralino dark matter density. When the $1 \sigma$
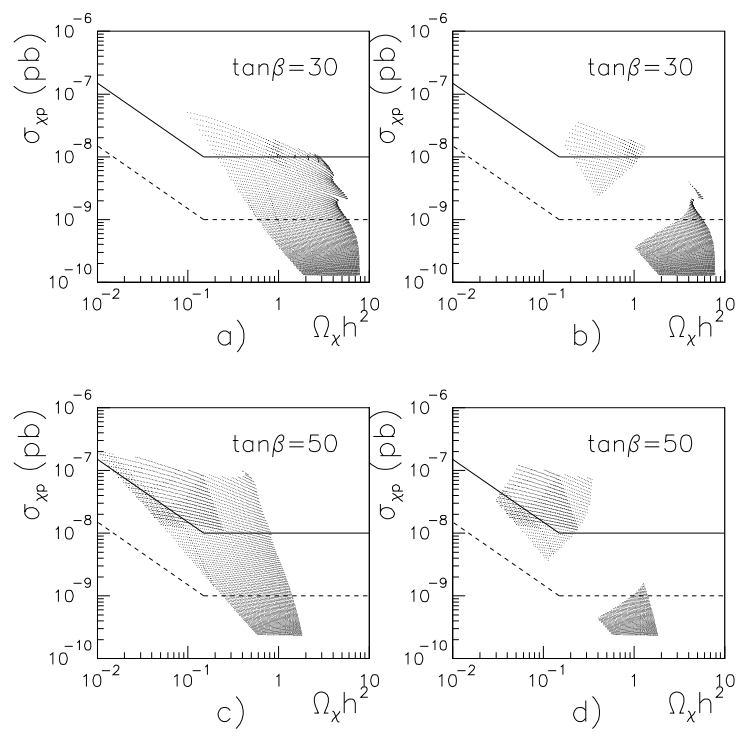

Figure 2: $\sigma_{\chi p}$ vs. $\Omega_{\chi} h^{2}$ in mSUGRA (a) without and (b) with $1 \sigma$ bound of $\Delta a_{\mu}$ for $\tan \beta=30$ respectively. The corresponding plots are shown in (c) and (d) for $\tan \beta=50$ respectively. We take $A=0$ and $\mu>0$, and scan $m \in[0,1] \mathrm{TeV}$ and $M \in[0,1] \mathrm{TeV}$, subject to experimental constraints. The straight and dashed lines show $\sigma_{\text {scaled }}=10^{-8}$ and $10^{-9} \mathrm{pb}$, the approximate level of the claimed sensitivity by the CDMS(Soudan) and GENIUS proposals respectively. We also indicate the region of parameter space where $\Delta a_{\mu}$ is more than $2 \sigma$ lower than current central value ((b) and (d) lower region).

bound of $\Delta a_{\mu}$ is imposed, there is not too much parameter space remaining for $\tan \beta \sim 10$. In Fig. 2(a) ((c)) and (b)((d)), we therefore show the allowed region of $\sigma_{\chi p}$ vs. $\Omega_{\chi} h^{2}$ without and with $\Delta a_{\mu}$ constraints for $\tan \beta=30(50)$ respectively. Here, we take $A=0$ and $\mu>0$ and allow $m$ and $M$ to vary in the intervals $m<1 \mathrm{TeV}$ and $M<1 \mathrm{TeV}$ and require $m_{h}>111 \mathrm{GeV}$, $m_{\chi^{ \pm}}>100 \mathrm{GeV}$ and neutral LSP. The straight and dashed lines show the approximate level of the claimed sensitivity by the CDMS(Soudan) 8] and GENIUS [10] proposal respectively. The calculation of $\sigma_{\chi p}$ is based on refs. [30, 44]. We use the value $m_{s}\langle p|\bar{s} s| p\rangle=130 \mathrm{MeV}$ for the strange quark's contribution to the nucleon mass; this matrix element is uncertain to about a 
factor of 2, leading to a similar uncertainty in the prediction of $\sigma_{\chi p}$. Finally, the calculation of the scaled LSP relic density $\Omega_{\chi} h^{2}$ uses results of refs. 43, 45]; $s$-channel poles are treated as described in ref. 46]. The co-annihilation of $\tilde{\chi}_{1}^{0}$ with sleptons is not included.

For $\tan \beta=30, \sigma_{\chi p}$ is larger than $\sim 3 \times 10^{-9}$ pb and can reach upto $\sim 4 \times 10^{-8}$ pb within $1 \sigma$ bound of $\Delta a_{\mu}$ (upper region in Fig. $2(\mathrm{~b})$ ). In this case, $\Omega_{\chi} h^{2}$ vary from $\sim 0.2$ to $\sim 1.6$. If we require a cosmological constraint $\Omega_{\chi} h^{2}<0.3$ [47], some portion of region are allowed and are within the approximate level of the claimed sensitivity by CDMS(Soundan) and the GENIUS proposal. Note that we do not include the $\tilde{\tau} \chi_{1}^{0}$ coannihilation here. The lower limit of $\Omega_{\chi} h^{2}$ might be reduced substantially for the points of most left-down portion of parameter space in Fig. 2 (a) and (b) where parameter space is ended due to the condition that $\chi_{1}^{0}$ is not LSP. Note however that the prediction is very sensitive to the running of the third generation sparticle $\tilde{\tau}$ between Planck scale and GUT scale [48, 49]. For $\tan \beta=50, \sigma_{\chi p}$ is larger than $\sim 4 \times 10^{-9} \mathrm{pb}$ and can reach up to $\sim 1 \times 10^{-7} \mathrm{pb}$ within $1 \sigma$ bound of $\Delta a_{\mu} . \Omega_{\chi} h^{2}$ is mostly less than 0.3 due to the effect of s-channel pseudoscalar Higgs pole [43, 31, 20] of neutralino pair annihilation and is thus cosmologically acceptable. The smaller value of $m_{A}$ is achived by the bottom Yukawa running of $m_{H_{d}}$ enhanced by $\tan ^{2} \beta$. Heavy higgs exchange contribution in $\sigma_{\chi p}$ is also enhanced as $H \bar{s} s$ coupling is enhanced and $m_{H}$ is reduced. Again all region is within the approximate level of the claimed sensitivity by the GENIUS proposal .

On the other hand, when $a_{\mu}$ turns out to be more than $2 \sigma$ below the current central value, $\sigma_{\chi p}$ is mostly below $1 \times 10^{-9} \mathrm{pb}$ and $\Omega_{\chi} h^{2}$ is larger than $1(0.4)$ for $\tan \beta=30(50)$. For $\tan \beta=10, \sigma_{\chi p}<0.5 \times 10^{-8} \mathrm{pb}$ and $\Omega_{\chi} h^{2}>0.4$ is found in our numerical study. They are cosmologically disfavored unless $\tilde{\tau} \tilde{\chi}_{1}^{0}$ coannihilation is taken into account.

\section{More general models}

Now we relax our assumptions, allowing for non-universal soft scalar masses at the GUT scale, while keeping the unification of the gaugino masses. As specific models, we consider a model with non-universal Higgs mass and an $S O(10)$ Grand Unified model [29].

In a model with non-universal Higgs, we assume that soft breaking Higgs mass terms are different from the other universal soft scalar mass terms at GUT scale,

$$
m \neq m_{H_{u}}\left(=m_{H_{d}}\right)
$$

For simplicity we keep $m_{H_{u}}=m_{H_{d}}$ at the GUT scale 1 .

The $S O(10)$ theory incorporates a complete generation of MSSM matter superfields into the 16-dimensional spinor representation, $\Psi_{16}$. In addition to these matter superfield, the minimal $S O(10)$ model includes a 10-dimensional Higgs superfield $\Phi_{10}$ which contains the two Higgs superfields of the MSSM (as well as their $S U(3)$ triplet, $S U(2)$ singlet partners). When $S O(10)$ breaks to the MSSM gauge group $S U(3)_{C} \times S U(2)_{L} \times U(1)_{Y}$, additional $D$-term contributions (parameterized by $M_{D}^{2}$ which can be either positive or negative) to the soft SUSY breaking masses arise [50]:

$$
\begin{array}{r}
m_{Q}^{2}=m_{E}^{2}=m_{U}^{2}=m_{16}^{2}+M_{D}^{2} \\
m_{D}^{2}=m_{L}^{2}=m_{16}^{2}-3 M_{D}^{2}, m_{H_{u, d}}^{2}=m_{10}^{2} \mp 2 M_{D}^{2},
\end{array}
$$

where $m_{16}$ and $m_{10}$ are scalar soft breaking masses for fields in the $\mathbf{1 6}$ and $\mathbf{1 0}$ dimensional representations of $S O(10)$, respectively. We consider this model too, but we assume $m_{16}=$ $m_{10} \equiv m$ for simplicity.

\footnotetext{
${ }^{3} \mathrm{~A}$ model with non-universal Higgs mass, eq. (7) can be considered as a special case of $S O(10)$ model, eq. (8) with $M_{D}^{2}=0$.
} 
The modifications (7) and (8) of the mSUGRA boundary conditions change our predictions through the changed value of $|\mu|$ and pseudoscalar Higgs mass $m_{A}$ at the weak scale, which mostly affects $\sigma_{\chi p}$ and $\Omega_{\chi} h^{2}$ (and also through modifications of the slepton spectrum, which change $\Delta a_{\mu}$ and $\Omega_{\chi} h^{2}$, for a $S O(10)$ model). The changes of $|\mu|$ value can be understood as follows. In the mSUGRA scenario, the contributions of $m$ and $M$ to the weak scale values of the soft breaking Higgs boson masses can be parameterized as

$$
\begin{gathered}
m_{H_{d}}^{2} \simeq m^{2}+0.5 M^{2} \\
m_{H_{u}}^{2} \simeq \epsilon_{H} m^{2}-3.3 M^{2},
\end{gathered}
$$

where we have assumed $\sin \beta \simeq 1$ but ignored contributions from the bottom Yukawa coupling. The coefficient $\epsilon_{H}$ is small, because the GUT scale value of $m_{H_{u}}^{2}$ is canceled by the scalar masses appearing in the RG running $t$. The effect of non-universality on $m_{H_{u}}^{2}$ can be parameterized by introducing $m_{Q}^{2}+m_{U}^{2}+m_{H_{u}}^{2}=3 m_{s}^{2}$ and $\delta m_{H}^{2}=m_{H_{u}}^{2}-m_{s}^{2}$ :

$$
m_{H_{u}}^{2}=\delta m_{H}^{2}+\epsilon_{H} m_{s}^{2}-3.3 M^{2} .
$$

This equation follows because the radiative correction to $m_{H_{u}}^{2}$ is proportional to $m_{Q}^{2}+m_{U}^{2}+$ $m_{H_{u}}^{2}$, hence the effect of RGE running is the same as in mSUGRA with the replacement $m^{2} \rightarrow m_{s}^{2}$. If $\sin \beta \simeq 1$, correct gauge symmetry breaking requires $\mu^{2} \simeq-m_{H_{u}}^{2}-M_{Z}^{2} / 2$, where all quantities are taken at the weak scale. In $\operatorname{mSUGRA}\left(\right.$ i.e., $\left.\delta m_{H}^{2}=0\right), \mu>M$ is predicted unless $m$ is extremely large. However, if $\delta m_{H}^{2}$ is non-zero, it directly affects the value of $|\mu|$ at the weak scale.

These modifications of the mSUGRA boundary conditions also have an impact on the Higgs masses. At tree level, the mass of the pseudoscalar Higgs boson is simply given by

$$
\begin{aligned}
m_{A}^{2} & =m_{H_{u}}^{2}+m_{H_{d}}^{2}+2 \mu^{2} \\
& \simeq m_{H_{d}}^{2}+\mu^{2}-M_{Z}^{2} / 2
\end{aligned}
$$

where all quantities are taken at the weak scale and in the second line, we used the relation $\mu^{2} \simeq-m_{H_{u}}^{2}-M_{Z}^{2} / 2$ which holds if $\sin \beta \simeq 1$. Therefore, $m_{A}^{2}$ depends on $m_{H_{d}}^{2}$ and $\mu^{2}$ at weak scale. When we increase the soft Higgs masses in same amounts, the increase of $m_{H_{d}}^{2}$ is compensated by the decrease of $\mu^{2}$ (through the increase of $m_{H_{u}}^{2}$ ). In the model with nonuniversal Higgs mass (with the assumption $m_{H_{u}}^{2}=m_{H_{d}}^{2}$ at GUT scale), $m_{A}^{2}$ thus does not change too much along the change of $m_{H} / m$. On the other hand, in the $S O(10)$ model, the modifications of $m_{H_{u}}^{2}$ and $m_{H_{d}}^{2}$ at GUT scale are in the opposite direction. In this model, $m_{A}^{2}$ would be quite reduced by the decrease of $m_{H_{d}}^{2}$ and $\mu$ when $M_{D}^{2}$ has a large and negative value.

The reduction of $m_{A}$ implies the reduction of charged Higgs mass $m_{H^{ \pm}}$and heaviest CP even Higgs mass $m_{H}$. This has a significant implication on $\Omega_{\chi} h^{2}, \sigma_{\chi p}$ and the branching ratio of $b \rightarrow s \gamma$ decay. In mSUGRA model, LSP pair annihilation through $\chi \chi \rightarrow A, H \rightarrow X$ channel is not important unless $\tan \beta \gg 1$. However, when $m_{A}$ and $m_{H}$ decrease, the above channel can be quite enhanced especially in the resonance region. This in turn gives quite reduced $\Omega_{\chi} h^{2}$. On the other hand, the neutralino-proton cross section $\sigma_{\chi p}$ increases as $m_{H}$ decreases. This is because the dominant contributions to $\sigma_{\chi p}$ comes from the exchange of the two CP-even Higgs bosons. Also $B R(b \rightarrow s \gamma)$ is affected by $m_{H}^{ \pm}$value through charged Higgs-top quark loop.

\footnotetext{
${ }^{4}$ The effective $\epsilon_{H}$ is slightly negative for low SUSY breaking scale, but turns positive if this scale is large. Recall that the relevant scale for the analysis of gauge symmetry breaking increases with increasing sparticle masses.
} 
The reduction of $m_{H}^{ \pm}$tends to increase $B R(b \rightarrow s \gamma)$ because the charged Higgs-top quark loop always interferes with SM contribution constructively. Therefore the preferred value of $\tan \beta$ is expected to be different from that of mSUGRA. Note that the ino-squark loop have a opposite sign to charged Higgs-top loop in mSUGRA model for $\mu>0$ case. For very large $\tan \beta$, they tend to overshoot the charged Higgs contribution.

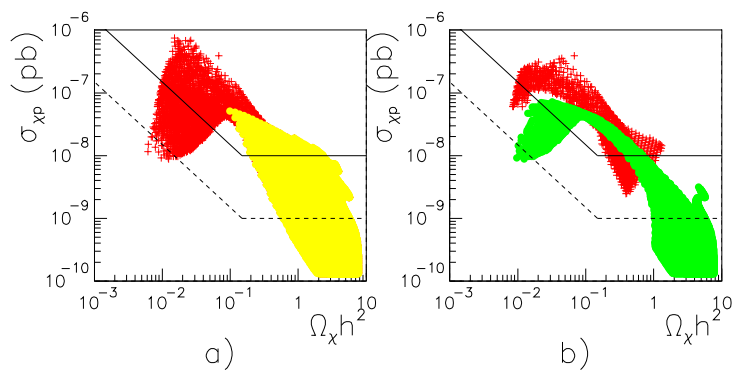

Figure 3: $\sigma_{\chi p}$ vs. $\Omega_{\chi} h^{2}$ in a model with non-unversal Higgs mass (a) without and (b) with $\Delta a_{\mu}$ constraint for $\tan \beta=30$ respectively. We take $A=0$ and $\mu>0$, and scan $m \in[0,1] \mathrm{TeV}, M \in[0,1] \mathrm{TeV}$ and $1 \leq m_{H_{u}}^{2} / m^{2} \leq 20$, subject to experimental constraints. The straight and dashed lines show the approximate level of the claimed sensitivity by the CDMS(Soudan) and GENIUS proposal respectively.

Fig. 3 shows the correlation between $\sigma_{\chi p}$ and $\Omega_{\chi} h^{2}$ for $\tan \beta=30, A=0$ and $\mu>0$ in a model with non-universal Higgs mass. Here, we scan the region $m<1 \mathrm{TeV}, M<1 \mathrm{TeV}$ and $1 \leq m_{H_{u}}^{2} / m^{2} \leq 20$, under the same mass constraint in Fig. 2. In Fig. 3(a), the light (yellow) shaded region indicates $m_{H_{u}}^{2} / m^{2}=1$, i.e, mSUGRA case. The maximum value of $\sigma_{\chi p}$ increases by about one order, compared to mSUGRA case and reaches up to $\sim 7 \times 10^{-7} \mathrm{pb}$ where $\Omega_{\chi} h^{2}$ value is about 0.02 and therefore LSP constitutes a subdominant part of local halo DM. The highest value of the scaled cross section, $\sigma_{\text {scaled }}$ is $\sim 10^{-7} \mathrm{pb}$. In Fig. 3(b), we further require that the $\Delta a_{\mu}$ constraint is satisfied. In this figure, the dark (red) shaded regions are correspond to the $1 \sigma$ bound case and the medium (green) shaded regions to the more than $2 \sigma$ below current central value case. For the $1 \sigma$ bound case, $\Omega_{\chi} h^{2}$ values in large portion of allowed regions are less than 0.3 and most of such regions are within the approximate level of the claimed sensitivity by the CDMS(Soudan) proposal. Also, for the $2 \sigma$ below case, the large portion of allowed region are cosmologically acceptable and within the sensitivity level by the GENIUS, contrary to mSUGRA model where all regions are cosmologically disfavored and have very small $\sigma_{\chi p}$ with $2 \sigma$ below case.

Fig. 4 shows the correlation between $\sigma_{\chi p}$ and $\Omega_{\chi} h^{2}$ for $\tan \beta=30, A=0$ and $\mu>0$ in $S O(10)$ model. Here, we scan the region $m<1 \mathrm{TeV}, M<1 \mathrm{TeV}$ and $-50000 \mathrm{GeV}^{2} \leq \mathrm{M}_{\mathrm{D}}^{2} \leq$ $+50000 \mathrm{GeV}^{2}$, under the same mass constraint in Fig. 2 f. In Fig. 4(a), the light (yellow)

\footnotetext{
${ }^{5}$ We keep all soft scalar masses are positive at GUT scale, therefore $-\frac{m^{2}}{2}<M_{D}^{2}<\frac{m^{2}}{3}$ always.
} 

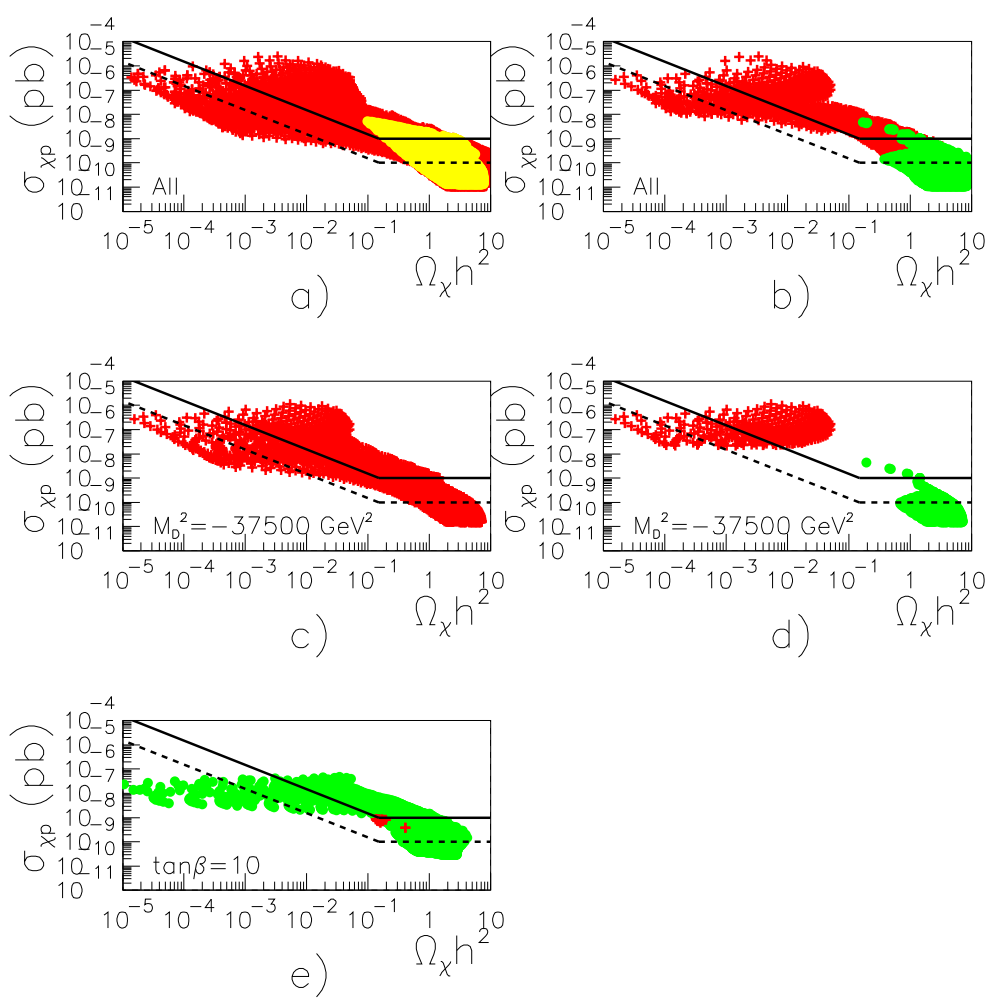

Figure 4: $\sigma_{\chi p}$ vs. $\Omega_{\chi} h^{2}$ in $S O(10)$ model (a) without and (b) with $\Delta a_{\mu}$ constraint for $\tan \beta=30$ respectively. We take $A=0$ and $\mu>0$, and scan $m \in[0,1] \mathrm{TeV}, M \in[0,1] \mathrm{TeV}$ and $-50000 \mathrm{GeV}^{2} \leq \mathrm{M}_{\mathrm{D}}^{2} \leq+50000 \mathrm{GeV}^{2}$, subject to experimental constraints. The corresponding plots are shown in (c) and (d) for fixed $M_{D}^{2}=$ $-37500 \mathrm{GeV}^{2}$. We also show the scatter plot in (e) with $\Delta a_{\mu}$ constraint for $\tan \beta=10$. The straight and dashed lines show the approximate level of the claimed sensitivity by the CDMS(Soudan) and GENIUS proposal respectively. 
shaded region indicates $M_{D}^{2}=0$, i.e, mSUGRA case. The maximum value of $\sigma_{\chi p}$ increases by more than two order of magnitude compared to mSUGRA case. It reaches up to $\sim 2 \times 10^{-5}$ $\mathrm{pb}$ where $\Omega_{\chi} h^{2}$ value is $\sim 4 \times 10^{-3}$. The maximum value of $\sigma_{\text {scaled }}$ is $\sim 2 \times 10^{-6} \mathrm{pb}$ 向 when $\Omega_{\chi} h^{2} \sim 2 \times 10^{-2}$. Therefore the LSP constitutes a subdominant part of the local halo DM in this case. In Fig. 4(b), we further require that $\Delta a_{\mu}$ bound is satisfied. In this figure, the dark (red) shaded regions are correspond to the $1 \sigma$ bound case. Large portion of the parameter space have cosmologically allowed $\Omega_{\chi} h^{2}<0.3$ and within the approximate level of the claimed sensitivity by the GENIUS proposal.

For $S O(10)$ model, the Higgs sector is changed drastically, as it can be read off directly from eq. (8). The heavy $\mathrm{CP}$ even and CP odd Higgs masses can be quite small if $M_{D}^{2}$ has large negative values. In this case, $\sigma_{\chi p}$ are largely enhanced and the LSP sometimes hit the Higgs pole leading to greatly reduced $\Omega_{\chi} h^{2}$. There appear the region where the detection rate (which depends on the product $\sigma_{\chi p} \Omega_{\chi}$ ) are enhanced by more than one order of magnitude, compared to mSUGRA and non-universal Higgs mass model, within cosmologically acceptable region. This happens when $m_{A} \ll 2 m_{\chi}$, therefore pole effect is reduced while $\sigma_{\chi p}$ is enhanced.

Such highest detection rate comes from the points where LSP is a subdominant component of the halo Dark Matter, as can be seen in the figure. For clarity, we show the correlation between $\sigma_{\chi p}$ and $\Omega_{\chi} h^{2}$ in Fig. 4 (c) without and (d) with $\Delta a_{\mu}$ constraints for $M_{D}^{2}=-37500$ $\mathrm{GeV}^{2}$.

The medium (green) shaded regions correspond to the parameter space where $a_{\mu}$ is lower more than $2 \sigma$ compared to the current central value. Here the effect of the existence of the $\mathrm{D}$ term is small; $\sigma_{\chi p}$ remains small and $\Omega_{\chi} h^{2}$ is too high. This is due to our artificial cut off of the parameter space of $M_{D}^{2}$. To make $\mu$ small to have acceptable $\Omega_{\chi} h^{2}$, one needs $M_{D}$ of roughly same order to that of gaugino masses. To compare we show the scatter plot for $\tan \beta=10$ in Fig. 4 (e). The SUSY scale is substantially smaller compared to in Fig. 4 (b) $(\tan \beta=30)$, therefore effect of $M_{D}^{2}$ is visible. The $2 \sigma$ below case could have acceptable $\Omega_{\chi} h^{2}$ and a large counting ratio for any value of $\tan \beta$ when $M_{D}^{2}$ is changed freely.

\section{Conclusions}

In this paper, we have investigated the implications of the recent measurement of muon MDM on the spin-independent neutralino-proton cross section $\sigma_{\chi p}$ and LSP dark matter relic density $\Omega_{\chi} h^{2}$ in several SUSY models with either universal or non-universal soft scalar masses at the GUT scale. We considered two extreme scenario for $\Delta a_{\mu}$ bound,i.e, $27 \times 10^{-10}<\Delta a_{\mu}<$ $59 \times 10^{-10}(1 \sigma$ bound $)$ and $0<\Delta a_{\mu}<11 \times 10^{-10}(2 \sigma$ below $) . \sigma_{\chi p}$ and $\Delta a_{\mu}$ can become large if $\tan \beta$ is large, because both quantities are sensitive to chirality violation in the matter (s)fermion sector (which in the MSSM is enhanced for large $\tan \beta$ ) and if the SUSY mass scale is low. On the other hand, $\Omega_{\chi} h^{2}$ become large if the SUSY mass scale is high. The $1 \sigma$ bound of $\Delta a_{\mu}$ prefer high $\tan \beta$ and low SUSY mass scale while for the $2 \sigma$ below case, low $\tan \beta$ and high SUSY mass scale are preferred. Therefore $1 \sigma$ bound of $\Delta a_{\mu}$ gives generally large $\sigma_{\chi p}$ and small $\Omega_{\chi} h^{2}$ while the opposite is true for $2 \sigma$ below. While these general statements are fairly model-independent as long as one sticks to the field content of the MSSM, quantitative predictions do depend significantly on details of the spectrum of superparticles, in particular on the implementation of radiative gauge symmetry breaking.

We considered three different models: mSUGRA, a model with non-universal Higgs mass, and an $S O(10)$ GUT model. In mSUGRA model, some portion of allowed parameter space

\footnotetext{
${ }^{6}$ This is the approximate level of claimed signal by DAMA[6].
} 
is cosmologically acceptable. The $\sigma_{\chi p}$ is within the sensitivity level by the GENIUS proposal, if we require $1 \sigma$ bound of $\Delta a_{\mu}$. On the other hand, if $\Delta a_{\mu}$ is $2 \sigma$ below the current central value, the maximum $\sigma_{\chi p}$ is mostly below $10^{-9} \mathrm{pb}$ and $\Omega_{\chi} h^{2}$ so large that it is cosmologically disfavored unless coannihilation of $\tilde{\tau} \chi_{1}^{0}$ is taken into account.

In a model with non-universal Higgs mass (for $1<m_{H_{u}}^{2} / m^{2}$ and $\tan \beta=30$ ), the maximum value of $\sigma_{\chi p}$ increases by about one order of magnitude while the minimum value of $\Omega_{\chi} h^{2}$ is reduce by about one order of magnitude. With the $1 \sigma$ bound of $\Delta a_{\mu}$, large portion of allowed parameter space gives cosmologically acceptable $\Omega_{\chi} h^{2}$ and are within the sensitivity level of CDMS(Soudan) proposal. Even with the case where $\Delta a_{\mu}$ is $2 \sigma$ below from current central value, some portion of parameter space gives cosmologically acceptable $\Omega_{\chi} h^{2}$ and are mostly within the sensitivity level of the GENIUS proposal. In this model, the maximal allowed value of dark matter detection rate (which depends on the product $\sigma_{\chi p} \Omega_{\chi}$ ) remains more or less same as that in mSUGRA model.

Dramatic deviations from mSUGRA predictions are possible if one introduces $S O(10)$ $D$-term contributions to scalar masses. In this model, within the $1 \sigma$ bound of $\Delta a_{\mu}$, the maximal allowed value of $\sigma_{\chi p}$ for given $\tan \beta$ can exceed the mSUGRA prediction by almost three order of magnitude, if the $D$-term contribution to the mass of the Higgs boson that couples to the top quark is positive and large. The corresponding $\Omega_{\chi} h^{2}$ is also reduced, however the counting ratio maybe enhanced by factor of 10 compared to the previous models. In this model, the higgs sector is changed drastically. The heavy CP even and CP odd Higgs masses can be quite small if $M_{D}^{2}$ has large negative values. The counting ratio is the highest when the LSP constitutes a subdominant potion of local halo DM.

The predictions of $\sigma_{\chi p}$ and $\Omega_{\chi} h^{2}$ depend on the boundary conditions of model parameters at GUT scale. The FCNC constraints only require universality in squark sector in the same gauge multiplets. The universality of sfermions and higgs masses at the GUT scale as in mSUGRA requires $\mu \gg M$, and it is not favored cosmologically. In mSUGRA model, only the parameter space with mass degeneracy of $\tilde{\tau}$ and $\chi_{1}^{0}$ or the $2 m_{\chi} \sim m_{A}$ is allowed.

The too large relic density may imply that LSP might have more higgsino component than predicted in the mSUGRA model, or equivalently non-universal scalar masses at the GUT scale for higgs sectors. The improved measurement of $\Delta a_{\mu}$, (non) discovery of dark matter at future search experiments, and improved observations of $\Omega_{\chi} h^{2}$ will give us a hint on the LSP natures. Especially, if DM is discovered in the early stage of near future experiments, we should consider the variation of the soft mass terms.

The implication to the collider physics is also important. Consistency to the cosmology force us to think about the parameter space where $\tan \beta \sim 50$ or $m_{\chi_{1}^{0}} \sim m_{\tilde{\tau}}$ in mSUGRA. For example, the degeneracy of stau and LSP mass indicates the domination of the decay mode into stau and very low acceptance of tau and anti-tau lepton pairs in $\chi_{2}^{0} \rightarrow \tilde{\tau} \tau$ decays at LHC. The end point of $m_{\tau \tau}$ in the cascade decay is important for the determination of sparticle masses [4]. However, it is equivalently possible that the nature's choice is a non-universal Higgs mass at GUT scale. In such case $\tilde{\chi}_{4}^{0}$ and $\tilde{\chi}_{2}^{+}$may have substantial wino component and therefore they will be frequently produced in $\tilde{q}_{L}$ decay at LHC. The identification of such decay modes and the determination of $\mu$ parameter is demonstrated in [51] for the case where SUSY scale is relatively low and the cascade decay of ino to slepton has substantial branching ratios. When SUSY scale is high, the ino with substantial higgino and gaugino mixing dominantly decays into Higgs bosons. The study for such a case might be interesting to see the ability for LHC to measure the relation between $\mu$ and $M$ to determine $\sigma_{\chi p}$ and $\Omega_{\chi} h^{2}$. 


\section{Acknowledgments}

We thank to S. Jhingan for careful reading of the manuscript. M.M.N. was supported in part by a Grant-in-Aid for Scientific Research from the Ministry of Education (12047217).

\section{References}

[1] For a review, see H. E. Haber and G. L. Kane, Phys. Rep. 117, 75 (1985).

[2] E. Witten, Nucl. Phys. B188, 513 (1981).

[3] J. Ellis, S. Kelley and D.V. Nanopoulos, Phys. Lett. B249, 441 (1990), and Phys. Lett. B260, 131 (1991); C. Giunti, C.W. Kim and U.W. Lee, Mod. Phys. Lett. A6, 1745 (1991); U. Amaldi, W. de Boer and H. Fürstenau, Phys. Lett. B260, 447 (1991); P. Langacker and M. Luo, Phys. Rev. D44, 817 (1991).

[4] See e.g. ATLAS Collab., ATLAS Detector and Physics Performance Technical Design Report. CERN/LHCC99-19. http://atlasinfo.cern.ch/Atlas/GROUPS/PHYSICS/TDR/access.htm; Proceedings of the "1996 DPF/DPB Summer Study on High-Energy Physics". Ed. D. G. Cassel, L. T. Gennari and R. H. Siemann; E. Accomando et al., [ECFA/DESY LC Physics Working Group Collaboration], Phys. Rep. 299, (1998), hep-ph/9705442.

[5] For a review, see G. Jungman, M. Kamionkowski and K. Griest, Phys. Rep. 267, 195 (1996).

[6] DAMA Collaboration, Phys. Lett. B480, 23 (2000).

[7] CDMS Collaboration, Nucl. Instrum. Meth. A444, 345 (2000), astro-ph/0002471.

[8] R. Schnee for the CDMS collab., talk at DARK 2000, Heidelberg, Germany, July 2000.

[9] CRESST Collaboration, Astropart. Phys. 12, 107 (1999), hep-ex/9904005.

[10] L. Baudis et al. Phys. Rep. 307, 301 (1998).

[11] H.N. Brown et al, "Precise measurement of the positive muon anomalous magnetic moemnt", hep-ex/0102017.

[12] A. Czarnecki and W. J. Marciano, hep-ph/0102122.

[13] L. Everett, G. L. Kane, S. Rigolin and L-T. Wang, hep-ph/0102145.

[14] J. L. Feng and K. T. Matchev, hep-ph/0102146.

[15] E. A. Baltz and P. Gondolo, hep-ph/0102147.

[16] U. Chattopadhyay and P. Nath, hep-ph/0102157.

[17] S. Komine, T. Moroi and M. Yamaguchi, hep-ph/0102204.

[18] J. Hisano and K. Tobe, hep-ph/0102315.

[19] T. Ibrahim, U. Chattopadhyay and P. Nath, hep-ph/0102324. 
[20] J. Ellis, D. V. Nanopoulos and K. A. Olive, hep-ph/0102331.

[21] R. Arnowitt, B. Dutta, B. Hu and Y. Santoso, hep-ph/0102344.

[22] K. Choi, K. Hwang, S. K. Kang, K. Y. Lee and W. Y. Song, hep-ph/0103048.

[23] J. E. Kim, B. Kyae and H. M. Lee, hep-ph/0103054.

[24] S. P. Martin and J. D. Wells, hep-ph/0103067.

[25] S. Komine, T. Moroi and M. Yamaguchi, hep-ph/0103182.

[26] S. Baek, P. Ko and H. S. Lee, hep-ph/0103218.

[27] The supersymmetric contribution to $a_{\mu}$ has previously been studied by numerous authors. A selection includes: J. Ellis, J. Hagelin and D.V. Nanopoulos, Phys. Lett. 116B, 283 (1982); J.A. Grifols and A. Mendez, Phys. Rev. D26, 1809 (1982); R. Barbieri and L. Maiani, Phys. Lett. B117, 203 (1982); D.A. Kosower, L.M. Krauss and N. Sakai, Phys. Lett. B133, 305 (1983); T.C. Yuan, R. Arnowitt, A.H. Chamseddine and Pran Nath, Z. Phys. C26, 407 (1984); H. König, Mod. Phys. Lett. A7, 279 (1992); U. Chattopadhyay and Pran Nath, Phys. Rev. D53, 1648 (1996), hep-ph/9507386; M. Carena, G.F. Giudice and C.E.M. Wagner, Phys. Lett. B390, 234 (1997), hep-ph/9610233.

[28] T. Moroi, Phys. Rev. D53, 6565 (1996); Erratum-ibid. D56, 4424 (1997), hepph/9512396.

[29] M. Drees, Y. G. Kim, T. Kobayashi and M. M. Nojiri, hep-ph/0011359.

[30] M. Drees and M.M. Nojiri, Phys. Rev. D48, 3483 (1993), hep-ph/9307208.

[31] M. Gomez and J. Vergados, hep-ph/0012020; M. Gomez, G. Lazarides and C. Pallis, Phys. Rev. D61, 123512 (2000); J. Ellis, T. Falk, G. Ganis, K. A. Olive and M. Srednicki, hep-ph/0102098; R. Arnowitt, B. Dutta and Y. Santoso, hep-ph/0102181.

[32] Early analyses of neutralino-nucleon scattering include: M.W. Goodman and E. Witten, Phys. Rev. D31, 3059 (1985); K. Griest, Phys. Rev. D38, 2357 (1988), Erratum-ibid. D39, 3802, (1989); M. Srednicki and R. Watkins, Phys. Lett. B225, 140 (1989); M. Kamionkowski, Phys. Rev. D44 3021 (1991); G.B. Gelmini, P. Gondolo and E. Roulet, Nucl. Phys. B351, 623 (1991); J. Ellis and R.A. Flores, Phys. Lett. B263, 259 (1991), and Phys. Lett. B300, 175 (1993).

[33] Recent analyses of neutralino-nucleon scattering in the MSSM include: H. Baer and M. Brhlik, Phys. Rev. D57, 567 (1998), hep-ph/9706509; E. Accomando, R. Arnowitt, B. Dutta and Y. Santoso, Nucl. Phys. B585, 124 (2000), hep-ph/0001019; J. Ellis, A. Ferstl and K.A. Olive, hep-ph/0007113, and Phys. Lett. B481, 304, (2000), hep-ph/0001005; V. Mandic, A. Pierce, P. Gondolo and H. Murayama, hep-ph/0008022; A.B. Lahanas, D.V. Nanopoulos and V.C. Spanos, hep-ph/0009065; A. Bottino, F. Donato, N. Fornengo and S. Scopel, hep-ph/0010203.

[34] J. Ellis et al., in ref. 31]

[35] F. J. Yndurain, hep-ph/0102312.

[36] G. Duda, G. Gelmini and P. Gondolo, hep-ph/0102200. 
[37] For a review, see e.g. L.E. Ibáñez and G.G. Ross, in Perspectives on Higgs physics, G.L. Kane, editor; hep-ph/9204201,

[38] M. Drees and M.M. Nojiri, Phys. Rev. D45, 2482 (1992).

[39] See e.g. http://lephiggs.web.cern.ch/LEPHIGGS/ .

[40] J.R. Espinosa and R.-J. Zhang, JHEP 0003, 026 (2000), hep-ph/9912236; M. Carena, H.E. Haber, S. Heinemeyer, W. Hollik, C.E.M. Wagner and G. Weiglein, Nucl. Phys. B580, 29 (2000), hep-ph/0001002.

[41] J. Ellis, G. Ganis, D. V. Nanopoulos and K. A. Olive, Phys. Lett. B502, 171 (2001), hep-ph/0009355.

[42] ALEPH collab., R. Barate et al., hep-ex/0011047.

[43] M. Drees and M. M. Nojiri, Phys. Rev. D47, 376 (1993).

[44] A. Djouadi and M. Drees, Phys. Lett. B484, 183 (2000), hep-ph/0004205.

[45] C. Boehm, A. Djouadi and M. Drees, Phys. Rev. D62, 035012 (2000), hep-ph/9911496. See also J. Ellis, T. Falk, K.A. Olive and M. Srednicki, Astropart. Phys. 13, 181 (2000), hep-ph/9905481.

[46] M. Drees and A. Yamada, Phys. Rev. D53, 1586 (1996), hep-ph/9508254.

[47] See e.g. J.R. Primack, talk at the 4th International Symposium on Sources and Detection of Dark Matter in the Universe (DM 2000), Marina del Rey, California, February 2000, astro-ph/0007187.

[48] R. Barbieri and L.J.Hall, Phys.Lett. B338, 212 (1994).

[49] Mihoko M. Nojiri, K. Fujii, T. Tsukamoto, Phys. Rev. D54, 6756 (1999)

[50] M. Drees, Phys. Lett. B181, 279 (1986); Y. Kawamura, H. Murayama and M. Yamaguchi, Phys. Rev. D51, 1337 (1995), hep-ph/9406245.

[51] M. Drees, Y.G. Kim, Mihoko M. Nojiri, D. Toya, K. Hasuko and T. Kobayashi, Phys. Rev. D63, 035008 (2001) 